\title{
The Occurrence of High Frequencies of MBL2 polymorphisms in the Xhosa ethnic group of South Africa
}

\author{
Inyang U. Udosen \\ Department of Genetics \& Biotechnology, Akwa Ibom State University, Mkpat-Enin, AKS, Nigeria
}

\begin{abstract}
Polymorphic changes in a given population give rise to genetic variations. These variations account for differences found within a population. One of the most abundant and common genetic variant that occur in the population is the Single Nucleotide Polymorphisms which are point mutations in genes. The study assessed the frequency of single nucleotide polymorphisms in mannose binding lectin (MBL2) gene namely rs8191996, rs 1838065 , rs 8179079 and rs1800450 in 366 adult individuals drawn from Xhosa ethnic group of South Africa. The polymorphisms showed high frequencies in its occurrence which was $0.71,0.42,0.71$ and 0.73 for rs8191996, rs1838065, rs 1800450 and rs8179079 respectively. Additionally, the genotype distribution of these polymorphisms showed a predominance of homozygotes over heterozygotes and were in accordance with Hardy-Weinberg principle. The high frequencies of the $M B L 2$ polymorphisms under study in this population suggest that there is a high incidence of single gene mutations in Xhosa ethnic group which could make the population vulnerable to infection.
\end{abstract}

DOI: $10.7176 / \mathrm{JBAH} / 10-6-04$

Publication date:March $31^{\text {st }} 2020$

\section{INTRODUCTION}

Single nucleotide polymorphisms (SNPs) are one of the forms of sequence variants that occur in the human genome but accounts for greater than 90\% of all differences found (Brumfield et al., 2003) and are the most abundant. SNPs are present $1-2 \%$ of the human genome which are found in every 300-600 nucleotides which imply that there are about 10 million SNPs present in the human genome (Kwiatkowski, 2000).

SNPs are useful molecular tools now used to study genes that might cause diseases- some SNP alleles cause disease by introducing difference in gene function or by regulation. However, many SNPs have little or no effect on disease but are useful markers in identifying marker SNP and functional SNP in disease association.

SNPs occur in intronic regions which contain splice sites leading to differences in the protein content of their exons. SNPs also occur in the promoter region of the gene and are reported to affect regulation and expression of proteins (Schork et al., 2000 and Prokunina et al., 2004). The combination of SNPs in structural genes and SNPs in the promoter region that controls its expression forms the haplotype of that gene. However, most SNPs occur in non-coding region of genes which can contain sequences that are important for regulatory functions (Cargill et al., 1999; Prokunina et al., 2004).

SNPs can change restriction enzyme recognition sites where alteration of a single base pair could lead to loss or gain of a restriction site. This is utilized in restriction fragment length polymorphisms (RFLPs). It is useful for cutting or digesting DNA fragments containing restriction site so as to distinguish alleles based on the product fragment sizes by electrophoresis (Schork et al., 2000; Prokunina et al., 2004).

Three missense mutations in exon 1 of mannose binding lectin $(M B L 2)$ are reported to be responsible for its deficiency (Presanis et al., 2003; Neth et al., 2000; and Worthley et al., 2005). They include mutation in codons 52 (allele D), codon 54 (allele B) and 57 (allele C)

Several reports have suggested that $\mathrm{C}$ allele of $M B L 2$ (codon 57) occurs at high frequency in sub-Saharan Africa while the B variant is reported to be found mainly in South America (Lipscombe et al.,1992; Madsen et al.,1998; Turner et al.,2000). Madsen and Co-workers (1998) reported that MBL2 genotypes from unrelated individuals in Mozambique population and Africans responsible for the low levels of the protein was codon 57 (C) variant occurring at a frequency of 0.24 while low concentrations of MBL in South America was due to extreme high allele frequency of codon 54 (B) variant in both Chiriguanos (0.42) and Mapuches (0.46). In this study, four MBL2 polymorphisms- rs8179079, rs1800450, rs8191996 and rs1838065- were utilized for the population screening of Xhosa adults.

\section{MATERIALS AND METHODS}

The sample population consisted of healthy black adults of 366 individuals drawn from Xhosa ethnic group. Blood samples were obtained in accordance with guidelines and approval from Ethics Committee of the Red Cross Hospital in Capetown Province. Genomic DNA was extracted from whole blood using QIAGEN DNA purification kit protocols. The SNPs used for MBL2 polymorphism study were rs8191996 (involving amino acid change in codon 176 of exon 4 by replacing asparagine with serine), rs 1838065 (occurring in intron 2 with no amino acid change), rs8179079 (having no amino acid change in codon 34 of exon 1) and rs1800450 (which 
change amino acid in codon 54 of exon 1 by replacing glycine with aspartic acid). Amplification Refractory Mutation System PCR was used to genotype $M B L 2$ polymorphisms which is a modified PCR technique (Newton et al., 1989)

Multiplex PCR was used for general screening of SNPs in the study population, with annealing temperature at $54^{\circ} \mathrm{C}$ and the proportion of the reagent were modified to increase its specificity by adding more $\mathrm{MgCl}_{2}$ to increase the activity of Taq polymerase at the total volume of $25 \mu \mathrm{l}$ as follows: $2.0 \mathrm{mM}$ of $\mathrm{MgCl}_{2}, 200 \mu \mathrm{M}$ of dNTPs, $0.25 \mu \mathrm{M}$ each for both forward and reverse primers, $4 \mathrm{U} / \mu \mathrm{l}$ of Taq polymerase, $4 \mathrm{U} / \mu \mathrm{l}$ of Taq Antibody and 20ng of Genomic DNA. The amplification reaction was carried out in Eppendorf MasterCycler Gradient PCR system. The primers used for multiplex PCR were 5'GAATGGAGCCATTCAGA G 3' (rs8191996G) and 5'GAATGGAGCATTCAGA A 3'(rs8191996A) as forward primers with 5'TATTATTGCTTTGTTGGTGCTGTT 3' as reverse primer for genotyping rs8191996 polymorphism; 5'CTCCCTGTAAACCCCAACCTA 3'(rs1838065A) and 5'CTCCCTGTAAACCCCAACCTG 3' (rs1838065G) as forward primers for rs 1838065 polymorphism with 5'TAAACGGGGTCCGTCCAATCT 3' as reverse primer; 5'GGAGAGCTACAGGCAATCACT 3' (rs8179079T) and 5'GGAGAGCTACAGGCAATCACC 3' (rs8179079C) as forward primers with 5'AGACACACCTGGGTTTCCACTCA 3' as reverse primer for rs8179079 polymorphism; 5'GGCAAAGATGGGCGTGATGG 3' $\quad$ (rs1800450G) and 5'GGCAAAGATGGGCGTGATGA 3' (rs1800450A) as forward primers for rs1800450 polymorphism along with the reverse primer 5'CATCCTGTGTCCACGGGATG 3'.

In the screening of the SNPs, four PCR reactions were carried out in two separate multiplex PCRs. In one multiplex PCR, the primers rs8191996A, rs1838065A, rs8179079T and rs1800450A were used. In the other multiplex PCR, the primers rs8191996G, rs1838065G, rs8179079T and rs1800450G were used.

The PCR products obtained were separated by subjecting them to electrophoresis in a $2 \%$ agarose gels stained with ethidium bromide and visualized under UV Gel Doc System for identification of the bands.

\section{RESULTS}

The 366 genomic DNA samples of black adult (Xhosa) population were used to screen for the occurrence of alleles of the MBL2 SNPs- rs8179079, rs1800450, rs8191996 and rs1838065 by multiplex PCR (figure 1).

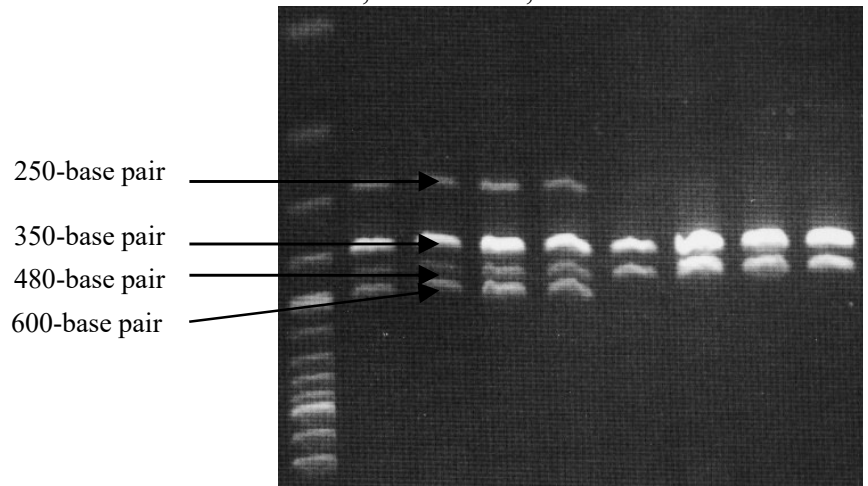

Figure1: Products of multiplex PCR showing rs1800450, rs8191996, rs817079 and rs1838065 showing 250, 350, 480 and 600-base pair respectively.

The genotype distribution of the MBL2 SNPs in the study population showed variations in the occurrence of alleles having a general predominance of homozygotes over heterozygotes (table 1). The rs8191996 SNP was found in 318 individuals with A allele occurring in 298(94\%) adults. Its allele frequency for A and G were found to be $477(75 \%)$ and $159(25 \%)$ respectively. Homozygote for the rs 8191996 variants accounted for 199 adults representing $62 \%$ as compared to the 119 adults (38\%) for heterozygosity.

\begin{tabular}{|l|l|l|l|}
\hline Polymorphism/ Genotype & rs8191996 & rs 1838065 & rs 1800450 \\
\hline AA & $179(56 \%)$ & $136(85 \%)$ & $20(6 \%)$ \\
\hline AG & $119(38 \%)$ & $11(7 \%)$ & $161(47 \%)$ \\
\hline GG & $20(6 \%)$ & $12(8 \%)$ & $161(47 \%)$ \\
\hline
\end{tabular}

Table1: Frequency of MBL genotypes in Adult Population.

The frequency of distribution for rs 1838065 in the adult population showed that they occurred in 159 individuals with the AA allele found in $136(85 \%)$ adults. The allele frequency for A and G allele was $283(89 \%)$ and $34(11 \%)$ respectively. $148(93 \%)$ of the adult controls were homozygote for rs 1838065 polymorphism.

MBL2 codon 54 variant SNP (rs1800450) was found in 342 individuals showing allelic frequency for A to be $201(29 \%)$ when contrasted with $483(71 \%)$ for G. The homozygote accounted for $181(53 \%)$ adults with the GG homozygote being more predominant which was found in 322 individuals.

In rs8179079 polymorphism, the variant SNP was observed in 320 individuals. The genotype frequencies were $196(61 \%), 103(32 \%)$ and 21 (7\%) adult controls for TT, CT and CC respectively. Allelic frequency was 
$145(23 \%)$ and $495(77 \%)$ for $\mathrm{C}$ and $\mathrm{T}$ allele respectively. The distribution of the variant in the adult population showed a predominance of homozygote above heterozygote accounting for 217 (61) individuals with the $\mathrm{T}$ allele being more predominant.

The genotypic analysis of the SNP alleles of rs8191996, rs1838065, rs1800450 and rs817079 in the population were in accordance with Hardy-Weinberg equilibrium.

The frequency of the MBL2 single nucleotide polymorphisms under study showed a general high occurrence of the gene mutations in Xhosa Adults with rs8191996, rs1800450 and rs8197079 at very high frequencies (table 2).

\begin{tabular}{|l|l|l|l|l|}
\hline Variant SNP & Wild Type & Heterozygous & Homozygous & Gene Frequency \\
\hline rs8191996 & 48 & 119 & 199 & 0.71 \\
\hline rs1838065 & 207 & 11 & 148 & 0.42 \\
\hline rs1800450 & 24 & 161 & 181 & 0.71 \\
\hline rs8179079 & 46 & 103 & 217 & 0.73 \\
\hline
\end{tabular}

Table 2: Occurrrence of $M B L 2$ gene mutations in the Xhosa Population

\section{DISCUSSION}

The rs1800450 MBL2 polymorphism which is the missense mutation occurring codon 54 of exon 1 of the gene is the allele B was to be found mainly in South America ((Lipscombe et al.,1992; Madsen et. al,1998; Turner et $a l ., 2000)$ and at a lesser frequency (0.11) in South African Coloured population (Hoal van Helden et al., 1999)). The allele B variant of $M B L 2$ also occur at very low frequency (0.03) in Namibian Bushmen (Hoal van Helden et $a l ., 1999)$. The result of this study disproves the notion that allele B variant does not occur in high frequency in any African population. This study showed that codon 54 variant (allele B) of $M B L 2$ occurs at a very high frequency of 0.71 in Xhosa ethnic group of South Africa which contradicts the study by Hoal Van Helden and Colleagues (1999) which showed that this mutation was non-existent in the Xhosa ethnic group. The reason for this discrepancy is due to the small population size (45) used by Hoal van Helden and Colleagues as opposed to 366 adults used in this study. The MBL2 polymorphisms under study- rs8179079, rs1800450, rs8191996 and rs1838065- were all in accordance with Hardy-Weinberg equilibrium which showed the predominance of homozygotes over heterozygotes. MBL polymorphisms especially codon 54 variant (allele B) along with codon 52 (allele D) and codon 57 (allele C) are known to predispose a population to infection due to its effect leading to low levels of MBL protein in the blood (Presanis et al., 2003; Neth et al., 2000; Worthley et al., 2005; Alves da Cruz et al., 2013 and Guo et al., 2017). Other MBL2 polymorphisms act by interacting with either allele B, C or D to influence predisposition to infections. Population screening of single nucleotide polymorphisms (SNPs) could be a useful tool for epidemiological study of genes that could predispose individuals in a population to diseases. Several genotyping technologies have been developed for SNPs which emphasizes its usefulness in pharmacogenomic and epidemiologic study.

\section{REFERENCES}

Alves da Cruz, H. L., da Silva, R.C., Segat, L., Carvalho, M. S., Brandao, A. C., Guimaraes, R. L.,Santos, F. C., Lira, A. S. Montenegro, L. M. L., Schindler, H. C., and Crovella, S. 2013, MBL2 gene polymorphisms and susceptibility to tuberculosis in a northeastern Brazilian population, Infection, Genetics and Evolution, 19: 323329

Brumfield, R. T., Beerli, P., Nickerson, D. A., and Edwards, S. V. 2003, The utility of Single nucleotide polymorphisms in inferences of population history, TRENDS in Ecology and Evolution, 18(5):249-256.

Cargill, M., Altshuler, D., Ireland, J., Sklar, P., Ardlie, K., Patil, N., Lane, C. R.,Lim, E. P., Kalyanaraman, N., Nemesh, J., Ziaugra, L., Friedland, L., Rolfe, A., Warrington, J., Lipshutz, R., Daley, G. Q., and Lander, E. S.1999, Characterization of single-nucleotide polymorphisms in coding regions of human genes, Nature Genetics 22:231-237.

Guo, Y. L., Liu, Y., Ban, W. J., Sun, Q. and Shi, G.L. 2017, Association of mannose-binding lectin gene polymorphisms with the development of pulmonary tuberculosis in China, Bio Med Central Infectious Diseases 17: 210

Hoal-van Helden, E. G., Epstein, J., Victor, T. C., Hon, D., Lewis, L., Beyers, N., Zurakowski, D., Ezekowitz, R. A. B. and van Helden, P. D. 1999, Mannose Binding Protein B Allele Confers Protection against Tuberculous Meningitis. Pediatr Res 45:459-464.

Kwiatkowski, D. 2000. Susceptibility to Infection. BMJ 321:1061-1064.

Lipscombe, R. J., Sumiya, M., Hill, A. V. S., Lau, Y. L., Levinsky, R. J., Summerfield, J. A. and Turner, M. W. 1992, High frequencies in African and non-African populations of independent mutations in the mannose binding protein gene. Human Molecular Genetics 1(9):709-715.

Madsen, H.O., Satz, M. L., Hogh, B., Sverjgaard, A. and Garred, P. 1998, Different Molecular Events Result in Low Protein Levels of Mannan-Binding Lectin Populations from Southeast Africa and South America. The 
Journal of Immunology. 3169-3175.

Neth, O., Jack, D. L., Dodds, A. W., Holzel, H., Klein, N. J. and Turner, M. W. 2000, Mannose-Binding Lectin Binds to a Range of Clinically Relevant Micro-organisms and Promotes Complement Deposition. INFECTION AND IMMUNITY 68(2):688-693.

Newton, C. R., Heptinstall, L. E., Powell, S. J., Summers, C., Kalsheker, N., Smith, J. C. and Markham, A. F. 1989, Analysis of any point mutation in DNA. The amplification refractory mutation system (ARMS) Nucleic Acids Research17(7):2503-2515.

Presanis, J. S., Kojima, M. and Sim, R. B. 2003, Biochemistry and genetics of mannan-binding lectin (MBL). Biochemical Society Transactions 31(4):748-752.

Prokunina, L. and Alarcon-Riquelme, M. E. 2004, Regulatory SNPs in complex diseases: their identification and functional validation. Expert reviews in molecular medicine 6(10):1-15.

Schork, N. J., Fallin, D. and Lanchbury, S. 2000, Single nucleotide polymorphisms and the future of genetic epidemiology. Clin. Genet. 58:250-264.

Tishkoff, S. A. and Williams, S. M. 2002, Genetic analysis of African populations: Human evolution and complex disease. Nature Reviews Genetics 3:611-621.

Turner, M. W., Dinan, L., Heatley, S., Jack, D. L., Boettcher, B., Lester, S., McCluskey, J. and Roberton, D. 2000, Restricted polymorphism of the mannose-binding lectin gene of indigenous Australians. Human Molecular Genetics 9(10):1481-1486.

Worthley, D. L., Bardy, P. G. and Mullighan, C. G. 2005, Mannose-binding lectin: biology and clinical implications. Internal Medicine Journal 35:548-555. 